\title{
Coordinated Analysis of Mercury Analog Samples Subjected to Simulated Space Weathering
}

Michelle Thompson ${ }^{1}$, Madison McGlaun ${ }^{1}$, Kathleen Vander Kaaden ${ }^{2}$, Mark Loeffler ${ }^{3}$ and Francis McCubbin $^{4}$

${ }^{1}$ Purdue University, West Lafayette, Indiana, United States, ${ }^{2}$ Jacobs/NASA Johnson Space Center, Houston, Texas, United States, ${ }^{3}$ Northern Arizona University, Flagstaff, Arizona, United States, ${ }^{4}$ NASA Johnson Space Center, Houston, Texas, United States

Introduction: The surfaces of airless bodies such as Mercury are continually modified by micrometeoroid impacts and solar wind irradiation, a process known as space weathering $[1,2]$. These interactions alter the microstructure, chemical composition, and optical properties of surface regolith. Spectral changes in surface regolith are driven by the production of submicroscopic particles, typically containing Fe. Our understanding of space weathering has largely been based on data from the Moon and near-Earth S-type asteroids. However, the space weathering environment at Mercury is much more extreme, experiencing more intense solar wind flux and higher velocity micrometeoroid impacts [3,4]. Additionally, the composition of Mercury's surface varies significantly from that of the Moon, including enrichments of up to $4 \mathrm{wt} . \% \mathrm{C}$, and low $(<2 \mathrm{wt} . \%)$ Fe [5,6]. Since Fe plays a critical role in the development of space weathering features on other airless surfaces, its limited availability may strongly affect the nature of space weathering on Mercury. To investigate how space weathering affects the chemical, microstructural, and optical properties of the surface of Mercury, we used pulsed-laser irradiation to simulate the short duration, high-temperature events associated with micrometeoroid impacts. For the experiments described here, we mixed forsteritic olivine (with varying $\mathrm{FeO}$ contents) with graphite and then performed reflectance spectroscopy, scanning electron microscopy (SEM) and transmission electron microscopy (TEM) to investigate the changes in these samples resulting from simulated space weathering.

Methods: We synthesized a series of forsteritic olivine samples with varying FeO contents at 1-bar at NASA Johnson Space Center (JSC) [7]. From these samples we prepared three pressed powder pellets for laser irradiation, mixing each olivine sample with 5 wt.\% graphite: 1) Sample SC-001, San Carlos olivine (9.1 wt.\% FeO), 2) Sample F-S-002 (0.05 wt.\% FeO), and 3) F-T-004 (0.53 wt.\% FeO) [7]. Samples were irradiated under vacuum at Northern Arizona University using a pulsed Nd-YAG laser, $(\lambda=1064 \mathrm{~nm}, \sim 6$ ns pulse duration). For each sample the laser was rastered 1x and then 5x over the surface. We collected in situ reflectance spectra from the surfaces of the sample after each raster using a Nicolet IS50 FourierTransform Infrared spectrometer (wavelength range of 0.65-2.5 $\mu \mathrm{m}$ ). We used an FEI Nova NanoSEM200 scanning electron microscope (SEM) at Purdue University to image the surface morphology and topography of the samples after irradiation. We prepared electron transparent thin section using an FEI Helios NanoLab 660 focused ion beam (FIB)-SEM at the University of Arizona. We analyzed the microstructural and chemical characteristics of the samples after laser irradiation using the $200 \mathrm{keV}$ scanning TEM at JSC equipped with an SDD $60 \mathrm{~mm}^{2}$ thin window energy dispersive X-ray spectrometer (EDX) and the $200 \mathrm{keV}$ Tecnai T20 TEM at Purdue University.

Reflectance Spectroscopy: The spectrum of the unirradiated SC-001sample exhibits a $1.0 \mu \mathrm{m}$ absorption feature, associated with $\mathrm{Fe}^{2+}$ in the olivine. After experiencing $5 \mathrm{x}$ laser shots, this absorption feature is much weaker. The unirradiated F-T-004 sample has a blue-sloped spectrum with low reflectance and no visible absorption features. With progressive laser irradiation, the reflectance of the sample increases and becomes strongly red-sloped. Finally, the unirradiated F-S-004 sample exhibits a dark, blue-sloped 
spectrum. After experiencing laser irradiation, the brightness of this sample increases significantly and the slope of the spectra for is slightly reddened compared to the unirradiated sample.

Scanning Electron Microscopy: There are two alteration textures observed in the samples exposed to pulsed-laser irradiation: 1) fluffy C-rich, and 2) vesiculated melt. The fluffy C-rich texture is evident in the 5x laser shot samples (Fig. 1a) and is composed of low-density deposits of C-rich material [8]. This microstructure is considerably different from the graphite we observe as unaltered at depth within the sample, comprised of discretely stacked layers. The second texture is a smooth and uniform melt layer distributed across isolated regions of the sample surface and contains vesicles ranging up to $100 \mathrm{~s}$ of $\mathrm{nm}$ in diameter (Fig. 1b).

Transmission Electron Microscopy: F-T-004 Melt Texture: This sample appears to contain a thin layer of amorphous melt material that coats the surface, $<100 \mathrm{~nm}$ thick (Fig. 2a). There are isolated regions which contain small nanoparticles, $<5 \mathrm{~nm}$ in diameter (Fig. 2a). EDX maps indicate the melt layer is depleted in oxygen compared to the underlying grains and may be enriched in silicon. SC-001 C-Rich Texture: This sample contains multiple rounded deposits measuring $\sim 1 \mu \mathrm{m}$ in diameter, discrete from stacked graphite, likely produced via melting from the laser. EDX maps reveal these deposits are enriched in $\mathrm{C}$ (Fig. 2b).

Discussion: The inclusion of both low-Fe minerals and graphite in these experiments create materials which are more analogous to the Mercurian surface than previous work [9]. Our results indicate that sample composition plays a significant role in space weathering on Mercury, exhibiting a strong correlation between spectral slope and Fe content. Variation in FeO content between the F-S-002 and FT-004 samples is < $0.6 \mathrm{wt}$.\% and yet the spectra deviate from flat to strongly reddened. The presence of very small nanoparticles, which may be Fe-bearing, are only observed in the FIB section containing melt extracted from F-T-004. It is likely that these nanoparticles are driving the strong spectral reddening observed in this sample. For the SC-001 sample, the fluffy C-rich textures may be developed by the amalgamation of small graphite particles into these unique morphologies. Such observations indicate that space weathering on Mercury may result in both familiar and new microstructural and chemical characteristics. 




Figure 1. Secondary electron (SE) images of the surface morphology of the a) F-S-002 sample showing fluffy C-rich textures, and the b) F-T-004 sample showing the vesiculated melt texture.

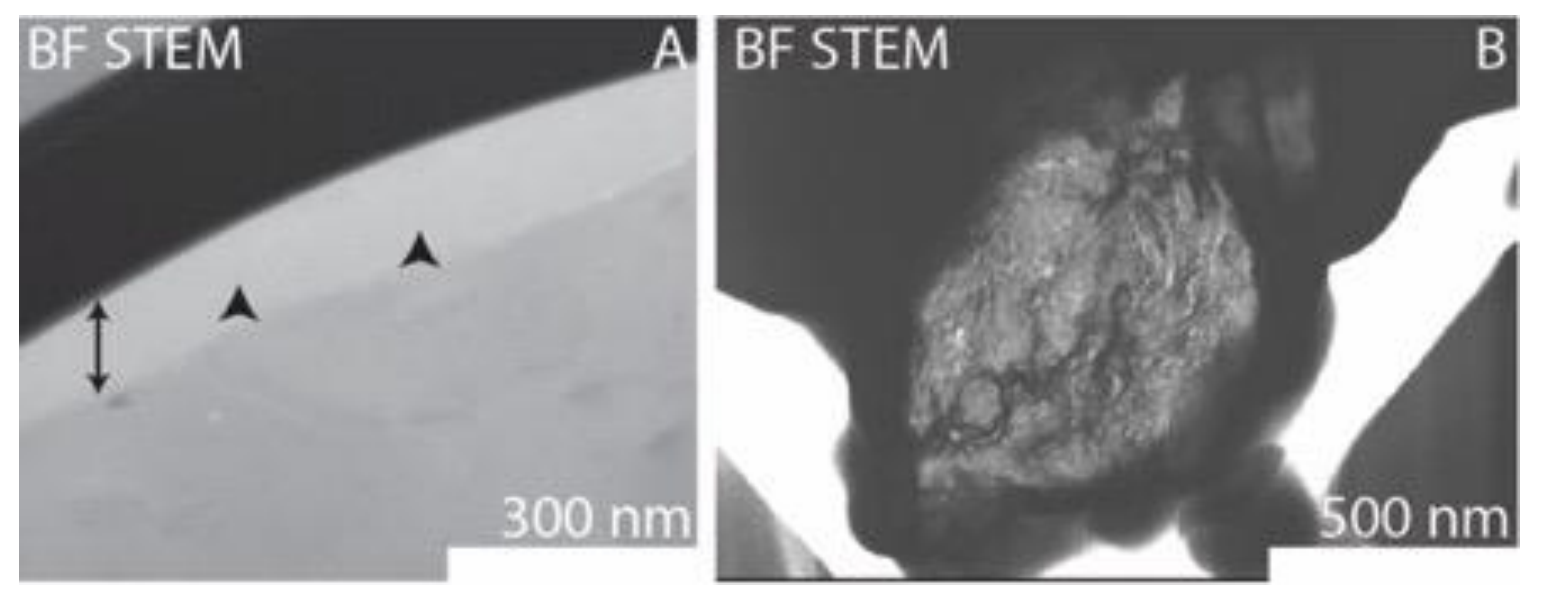

Figure 2. Bright field (BF) scanning TEM (STEM) images of a) the melt layer, about $100 \mathrm{~nm}$ in thickness in sample F-T-004. Very small $(<5 \mathrm{~nm})$ nanoparticles indicated by black arrows. B) BF STEM image of the melted $\mathrm{C}$ globule from the SC-001 sample. 


\section{References}

[1] Hapke B. (2001) J. Geophys. Res.-Planet., 106, 10039-10073.

[2] Pieters C.M. and Noble S.K. (2016) J. Geophys. Res-Planet., 121, 1865-1884.

[3] Lucey P.G., and Riner, M.A. (2011) Icarus, 212, 451-462.

[4] Cintala M.J. (1992) J. Geophys. Res.-Planet., 97, 947-973.

[5] Klima R.L. et al. (2018) Geophys. Res. Letters, 45, 2945-2953.

[6] Nittler L.R., et al. (2011) Science 333, 1847-1850.

[7] Vander Kaaden K.E., et al. (2018) LPSC XLIX, Abstract 1230.

[8] McGlaun M.L. et al. (2019) LPSC L, Abstract 2019.

[9] Trang D. et al. (2018) LPSC XLIX, Abstract 2083. 